\title{
A Combinatorial Approach to Obtain the Yield Probability Distribution along a Linearly-Loaded Cantilever Beam
}

\author{
Rasha A. Ali* \\ Muthanna A. Ali** \\ *College of Physical Education and Sports Science,University of Baghdad \\ **Department of Mechanical Engineering,University of Baghdad
}

Received 22/ 2/2016

Accepted 9/ 5/2016

(1) $9(9$

$\mathrm{EY}$ No ND This work is licensed

under a Creative Commons Attribution-NonCommercialNoDerivatives 4.0 International Licens

\begin{abstract}
:
The substantial key to initiate an explicit statistical formula for a physically specified continua is to consider a derivative expression, in order to identify the definitive configuration of the continua itself. Moreover, this statistical formula is to reflect the whole distribution of the formula of which the considered continua is the most likely to be dependent. However, a somewhat mathematically and physically tedious path to arrive at the required statistical formula is needed.

The procedure in the present research is to establish, modify, and implement an optimized amalgamation between Airy stress function for elastically-deformed media and the multi-canonical joint probability density functions for multivariate distribution completion, so that the developed distribution is to exhibit a sophisticated illustration of yield probability distribution along a cantilever beam whose structure is subjected to a linearly-distributed load. This combinatorial approach is to clarify the intensity of the stresses exerted onto the beam, to standardize the terms of stresses and their affection and to convert them into a more significant depiction of a probability distribution.
\end{abstract}

Key words: Multivariate Joint Probability Density Functions, Multi-Canonical Probability Functions, Airy Stress Function, Stress Analyses, Yield Probability Functions.

\section{Introduction:}

What is an intriguing to illustrate the sophisticated correlations between statistical distributions and engineering applications, which are widely spread in the present scientific worldwide prosperity? Particularly, engineering theories and applications may be clearly elucidated as soon as they are correlated with the corresponding statistical hypotheses, expectations, and/or probability distributions, which tend to

further clarify the conclusive expressions of these engineering applications. Therefore, the vast majority of the scientific, engineering and technological applications have the appreciable trend to arrive at their final expressions significantly tied to a probability distribution and/or hypothesis, which can further establish a comprehensible definition to these applications. To particularly study the 
continua considered in the present research, two-dimensional problems of elasticity may be effectively solved by the Airy stress function, or other stress functions, in order to arrive at the stresses' formulae. These stresses may be thereafter presented as a distribution along one or more specified axes. Afterwards, the stress distribution may be converted into a one-variable or a multivariate probability distribution, so that it can further be developed into a pellucid perspective to describe its magnitudes. Generally, each physical phenomenon is directly related to a mathematical and/or statistical interpretation with the fact of being dependently solved by each other.

D. Yevick (2003) [1] exhibits that it is convenient to evaluate the joint probability density functions identically. Afterwards, the joint probability density functions can then be calculated between the polarization mode dissemination's first and second orders in optical fibers.

$$
\text { C. H. Kim (2010) }
$$
demonstrates that the non-linear largedeflection-state stress distribution's Airy stress function in a simply-supported plate, with movable edges, can be determined by the use of the superposition technique of the Airy stress functions for an isotropic condition, with which the movable edges' boundary conditions and the state of the large deformation are satisfied.

In the present research, a combined method of Airy stress function methodology, to evaluate the stress field, and a developed multicanonical evaluation by the joint probability density functions is presented for a cantilever solid beam, which is linearly loaded along its total span. Charts for the stress field and the yield probability distribution, for multiple values of beam length and breadth, is to be thereafter obtained in order to recognize the points within which the yielding phenomenon is the most likely to happen.

\section{The Combinatorial Approach:}

Before presenting the medium considered mathematically, it is significant to count on assuming that the loaded beam is isotropic, i.e. every point within which has the same physical, thermal and mechanical properties. Furthermore, it is convenient to assume that the developed stresses only emanate in the $x y$-plane, so that there are no rules to abide regarding to evaluate the $z$-axis stresses and hence yielding probabilities, as well as the assumption which is based on the fact that all the developed stress field is emanated elastically, in such a way the twodimensional Hooke's law, compatibility, and Airy stress function relation are adequately applicable.

Referring to Fig. (1), the isotropic cantilever beam, having a length $L$ and a breadth $a$, is linearly loaded so that the point $(0, a / 2)$ is free from the distributed load and the point $(L, a / 2)$ is subjected to a distributed load whose value is $q L$.

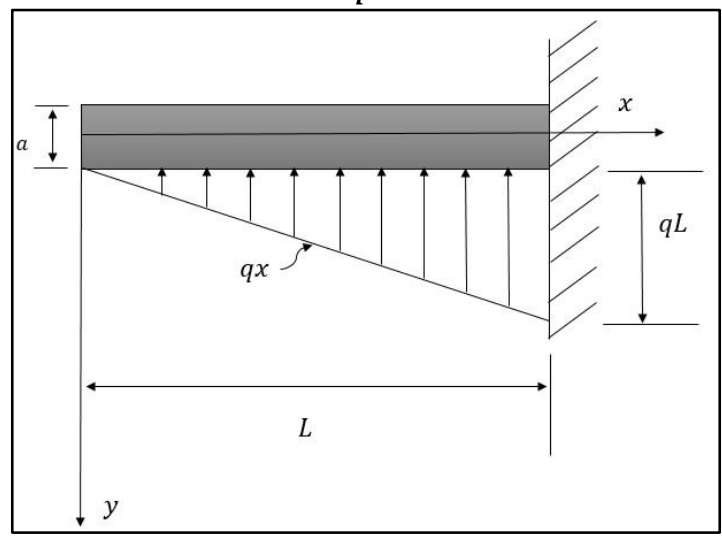

Fig. (1) The Mathematical Model [3]

From the geometry of Fig. (1), the boundary conditions can only be represented in the following equation(s) 


$$
\begin{gathered}
\sigma_{x x}(0, \pm y)=0 \\
\sigma_{y y}\left(0, \pm \frac{a}{2}\right)=0 \\
\sigma_{y y}\left(x,+\frac{a}{2}\right)=q x \\
\tau_{x y}\left(x, \pm \frac{a}{2}\right)=0 \\
\int_{-a}^{a} \tau_{x y}(0, y) d y=0
\end{gathered}
$$

The stress field relations can only be written in terms of the Airy stress function, being differentiated with respect to $x$ and/or $y$ axes, as the following formula shows [2]

$$
\left[\begin{array}{c}
\sigma_{x x} \\
\sigma_{y y} \\
\tau_{x y}
\end{array}\right]=\left[\begin{array}{c}
\partial^{2} \Phi / \partial y^{2} \\
\partial^{2} \Phi / \partial x^{2} \\
\partial^{2} \Phi / \partial x y
\end{array}\right] \ldots(2)
$$

Also, the following bi-harmonic equation must be satisfied in order to establish the appropriate mathematical and geometrical interpretation of the cantilever beam

$\nabla^{4} \Phi=0$

Assuming that the Airy stress function is of the sixth order which has 25 constants, this may be illustrated in the following relation in terms of the $x$ and $y$ variables

$$
\begin{aligned}
\Phi=A_{1} x^{2}+ & A_{2} x y+A_{3} y^{2}+A_{4} x^{3} \\
& +A_{5} x^{2} y+A_{6} x y^{2} \\
& +A_{7} y^{3}+A_{8} x^{4}+A_{9} x^{3} y \\
& +A_{10} x^{2} y^{2}+A_{11} x y^{3} \\
& +\cdots+A_{25} y^{6} \ldots(4)
\end{aligned}
$$

After mathematically combining the equations (1), (2), (3) and (4) in order to solve the constants which are needed to arrive at the stress field, this will lead into the following expression for the stresses induced within the cantilever beam [3]

$$
\begin{aligned}
& {\left[\begin{array}{l}
\sigma_{x x} \\
\sigma_{y y} \\
\tau_{x y}
\end{array}\right]} \\
& =\left[\begin{array}{l}
\frac{q x^{3} y}{4 a^{3}}+\frac{q}{4 a^{3}}\left(-2 x y^{3}+\frac{6}{5} a^{2} x y\right) \\
-q \frac{x}{2}+q x\left(\frac{y^{3}}{4 a^{3}}-\frac{3 y}{4 a}\right) \\
\left(\frac{3 q x^{2}}{8 a^{3}}+\frac{3 q}{20 a}\right)\left(a^{2}-y^{2}\right)-\frac{q}{8 a^{3}}\left(a^{4}-y^{4}\right)
\end{array}\right]
\end{aligned}
$$

Now, it is appropriate to combine the definition of the three yield probabilities $f(x, y)$ [1] [4], with which one can determine whether the beam yields, with the stresses in terms of the distributed load intensity $q$ (which equals to $0.001 \mathrm{MPa}$ ), the breadth $a$, the length $L$, and the variables $x$ and $y$, so that this will lead to the following final expression [5] [4].

$$
\begin{aligned}
& \frac{f_{x x}(x, y)=}{(2 \pi)^{\frac{3}{2}} \sigma_{x x} \sigma_{y y} \tau_{x y}\left(1-r^{2}\right)^{1 / 2}} \exp \left[-\frac{1}{2}\left(\frac{w}{\sigma_{x x}}\right)^{2}\right] * \\
& \exp \left[\frac{1}{2\left(1-r^{2}\right)}\left(\frac{w}{\sigma_{y y}}\right)^{2}+\left(\frac{w}{\tau_{x y}}\right)^{2}-\right. \\
& \left.2 r\left(\frac{w^{2}}{\sigma_{y y} \tau_{x y}}\right)\right] \ldots \ldots(6) \\
& f_{y y}(x, y)= \\
& \frac{1}{(2 \pi)^{\frac{3}{2}} \sigma_{x x} \sigma_{y y} \tau_{x y}\left(1-r^{2}\right)^{1 / 2}} \exp \left[-\frac{1}{2}\left(\frac{w}{\sigma_{x x}}\right)^{2}\right] * \\
& \exp \left[\frac{1}{2\left(1-r^{2}\right)}\left(\frac{w}{\sigma_{y y}}\right)^{2}+\left(\frac{w}{\tau_{x y}}\right)^{2}-\right. \\
& \left.2 r\left(\frac{w^{2}}{\sigma_{y y} \tau_{x y}}\right)\right] \ldots \ldots(7)
\end{aligned}
$$

$f_{x y}(x, y)=$

$\frac{1}{(2 \pi)^{\frac{3}{2}} \sigma_{x x} \sigma_{y y} \tau_{x y}\left(1-r^{2}\right)^{1 / 2}} \exp \left[-\frac{1}{2}\left(\frac{w}{\sigma_{x x}}\right)^{2}\right] *$ $\exp \left[\frac{1}{2\left(1-r^{2}\right)}\left(\frac{w}{\sigma_{y y}}\right)^{2}+\left(\frac{w}{\tau_{x y}}\right)^{2}-\right.$

$\left.2 r\left(\frac{w^{2}}{\sigma_{y y} \tau_{x y}}\right)\right]$

Provided that, 


$$
\begin{aligned}
& w=1 / 2\left(a+L \sqrt{\frac{1-x}{y}}\right)\left(1-\frac{x y}{\sqrt{4+y}}\right) \\
& r=1 / 2\left(a+L \sqrt{\frac{1-y}{x}}\right)\left(1-\frac{x y}{\sqrt{4+x}}\right)
\end{aligned}
$$

in such a process that these yield probabilities satisfy the following multivariate joint probability density function basic condition

$$
\int_{-\infty}^{\infty} f_{x x}(x, y) d x d y=1 \ldots(10)
$$

\section{Analyses of the Results:}

It has been previously demonstrated that the plane stress components, $\sigma_{x x}, \sigma_{y y}$, and $\tau_{x y}$, are explicitly related to their corresponding yield probabilities $f_{1}, f_{2}$, and $f_{3}$, which represent $f_{x x}, f_{y y}$, and $f_{x y}$ in the equations 6,7 , and 8 respectively, so that the present combinatorial analysis will importantly pose a new illustrative approach to indicate how the beam yielding process will be and which point(s) will be the most likely to undergo yielding.

The $x$-axis stresses $\sigma_{x x}$ have been demonstrated, referring to the Figures (2), (5) and (8), that the points $\left(L, \pm \frac{a}{2}\right)$ have their maximum decreased gradually as values of $y$ decrease from $\pm \frac{a}{2}$ to 0 , and $\sigma_{x x}$ values are also prone to a recognizable decay when $x$ values fall from $L$ until they become 0 for all values of the length $L$ and the breadth $a$. Furthermore, $\sigma_{x x}$ values noticeably augment when $a$ and/or $L$ increase. Whereas the $y$-axis stresses $\sigma_{y y}$ are found at their maximal magnitudes at $\left(L, \frac{a}{2}\right)$ for all values of $a$ and $L$. Also, $\sigma_{y y}$ values increase when the values of $x, y, a$ and/or $L$ increase as shown in Figures (3), (6), and (9). On the other hand, the $x y$-plane shear stresses $\tau_{x y}$ have their maximums in the point $(x, 0)$, and their minimums in the point(s) $\left(x, \pm \frac{a}{2}\right)$ for all values of $x, a$ and $L$, the values of $\tau_{x y}$ also increase when $x, a$, and/or L increase as shown in Figures (4), (7), and (10).

Now, the idea of combining the stresses' effects with the joint probability density functions has become, in many engineering and statistical aspects, significantly substantial so that it further furnishes prestigiously illustrative methods to investigate the percentages of the stress effects statistically upon the solid beam structure. To illustrate, the three yield probabilities $f_{1}, f_{2}$ and $f_{3}$ effects are to be discussed in terms of the variables $x, y, a$, and $L$, although the fact that the percentages of the three yield probabilities appear independent of the values of both $a$ and $L$. Firstly, it is clear that about 53.5 percent the $\mathrm{x}$ axis yield probability $f_{1}$ according to which the beam, for all the values of $y$, will likely to yield at the line $x=L$, while other lines $x=0, x=0.2 L, x=$ $0.4 L, x=0.6 L$ and, $x=0.8 L$

exhibit $0,0.5,3.5,12$ and 30.5 percent of $f_{1}$ respectively. Therefore, it appears that the beam yield point starts from the line $x=L$, and it will, depending on the intensity and the nature of the distributed load, continuously move until arriving at the line $x=0$. Secondly, about 33 percent is the $y$-axis yield probability $f_{2}$ at $x=L$, at which the beam will start yielding until it reaches $x=0$, with the possible plastic deformation and collapse if the distributed load increases. In addition, the other lines of constant values of the $y$-axis $x=0, x=0.2 L, x=0.4 L, x=$ $0.6 \mathrm{~L}$ and, as done, $x=$ $0.8 L$ exhibit $0,7,13,20$ and 27 percent of $f_{2}$ respectively. Thirdly, the $x y$-plane shear yield probability $f_{3}$ is obviously equal to 45 percent at the line $x=L$, which tends towards yielding the beam so that it helps the yielded zone keep moving to the other points until the 
line $x=0$ is reached. Moreover, the other assumed lines $x=0.2 L, x=$ $0.4 L, x=0.6 L$ and, so do other specified lines, $x=0.8 \mathrm{~L}$ exhibit $0,1,8,16$ and 30 percent of the $x y$ plane yield probability respectively. As previously mentioned, the sum of each probability for all values of $x$ satisfy the equation (10) [6]. Figures (11) to (19) illustrate the values, as percentages of stacked bars, of the yield probabilities $f_{1}, f_{2}$ and $f_{3}$.

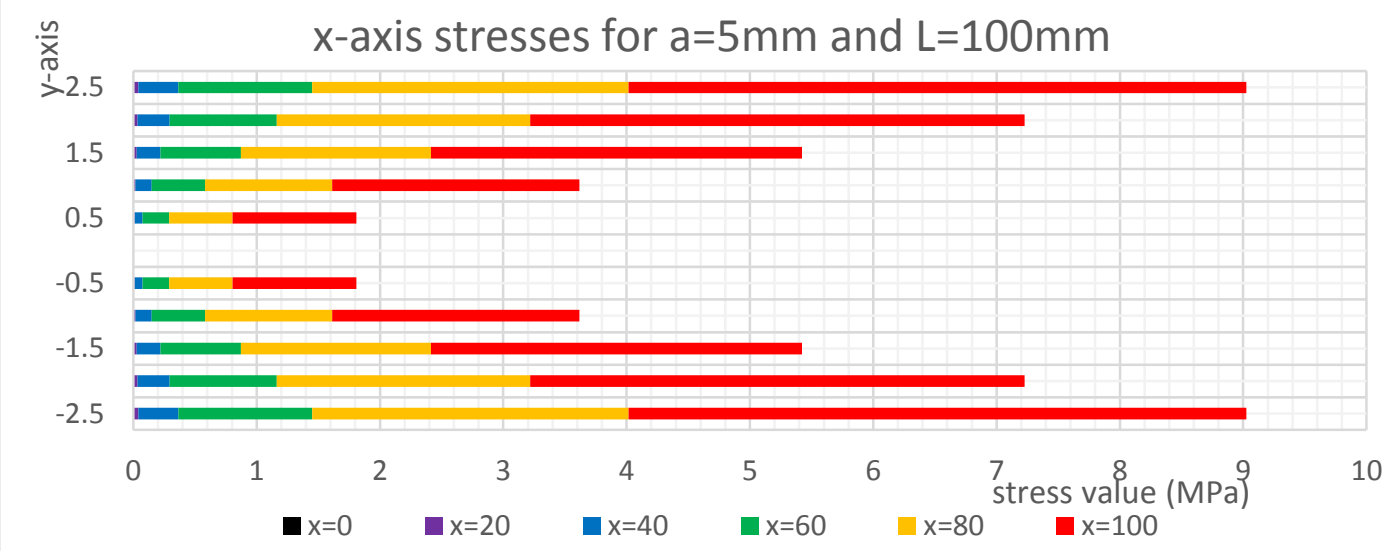

Fig. (2) $X$-Axis Stresses for $a=5 \mathrm{~mm}$ and $L=100 \mathrm{~mm}$

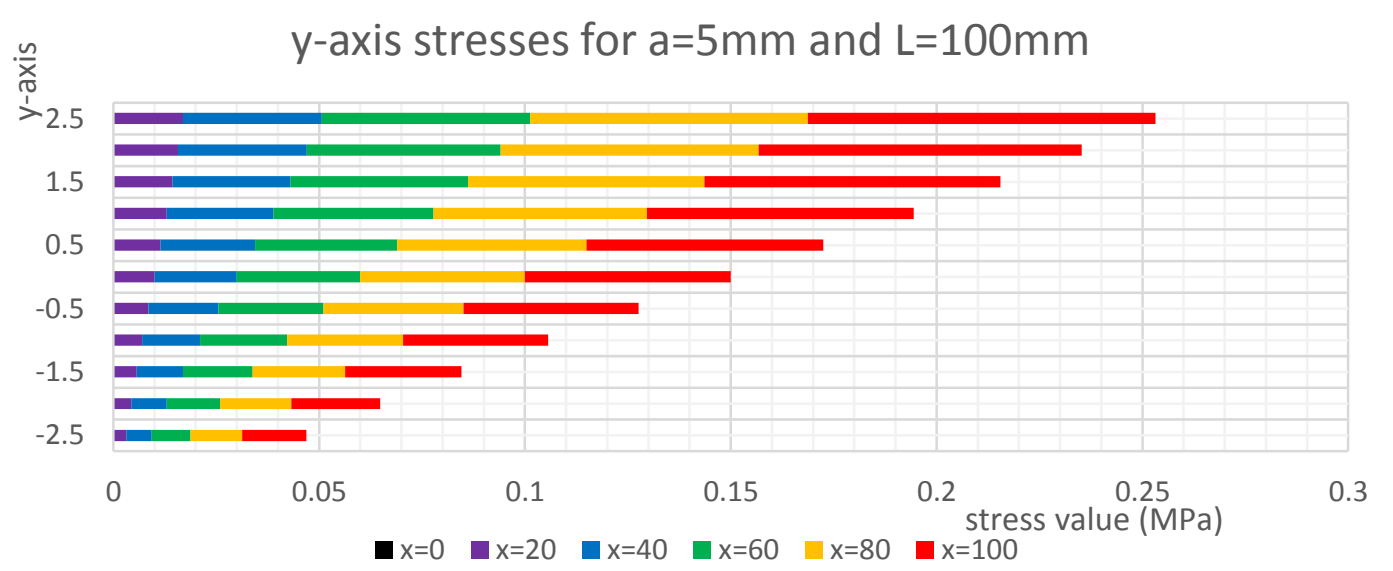

Fig. (3) Y-Axis Stresses for $\mathrm{a}=5 \mathrm{~mm}$ and $\mathrm{L}=100 \mathrm{~mm}$

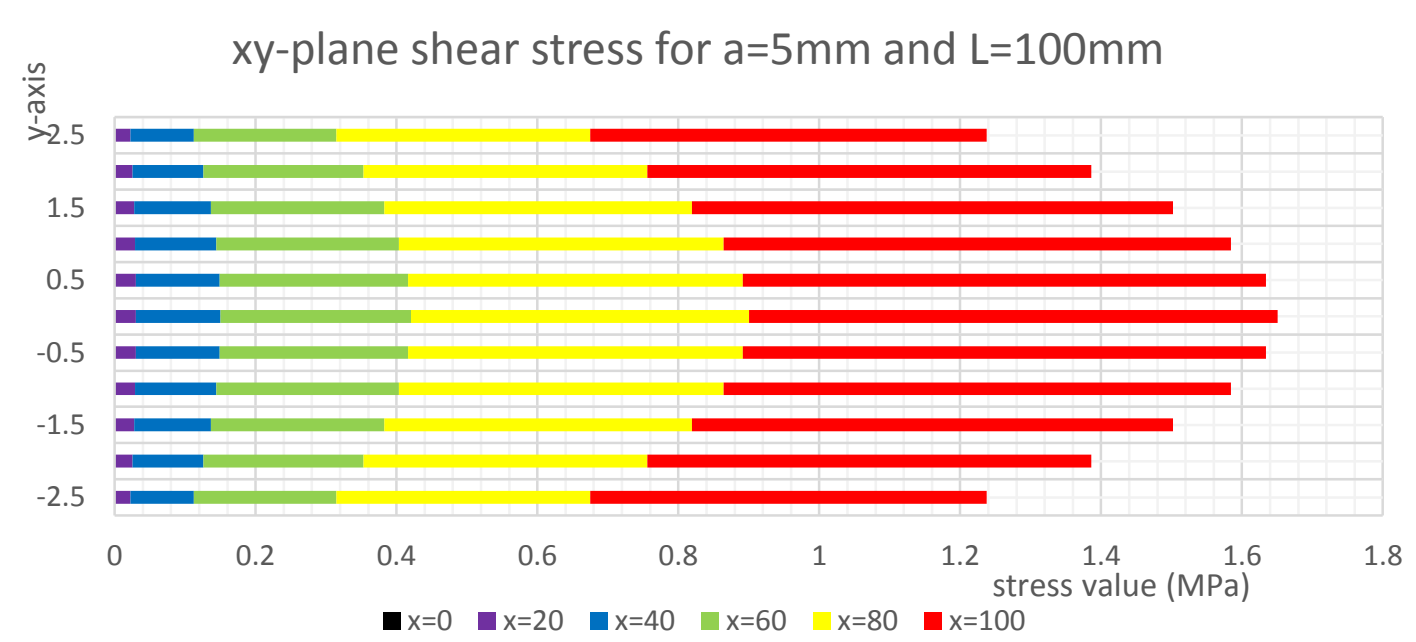

Fig. (4) XY- Plane Shear Stress for $a=5 \mathrm{~mm}$ and $L=100 \mathrm{~mm}$ 


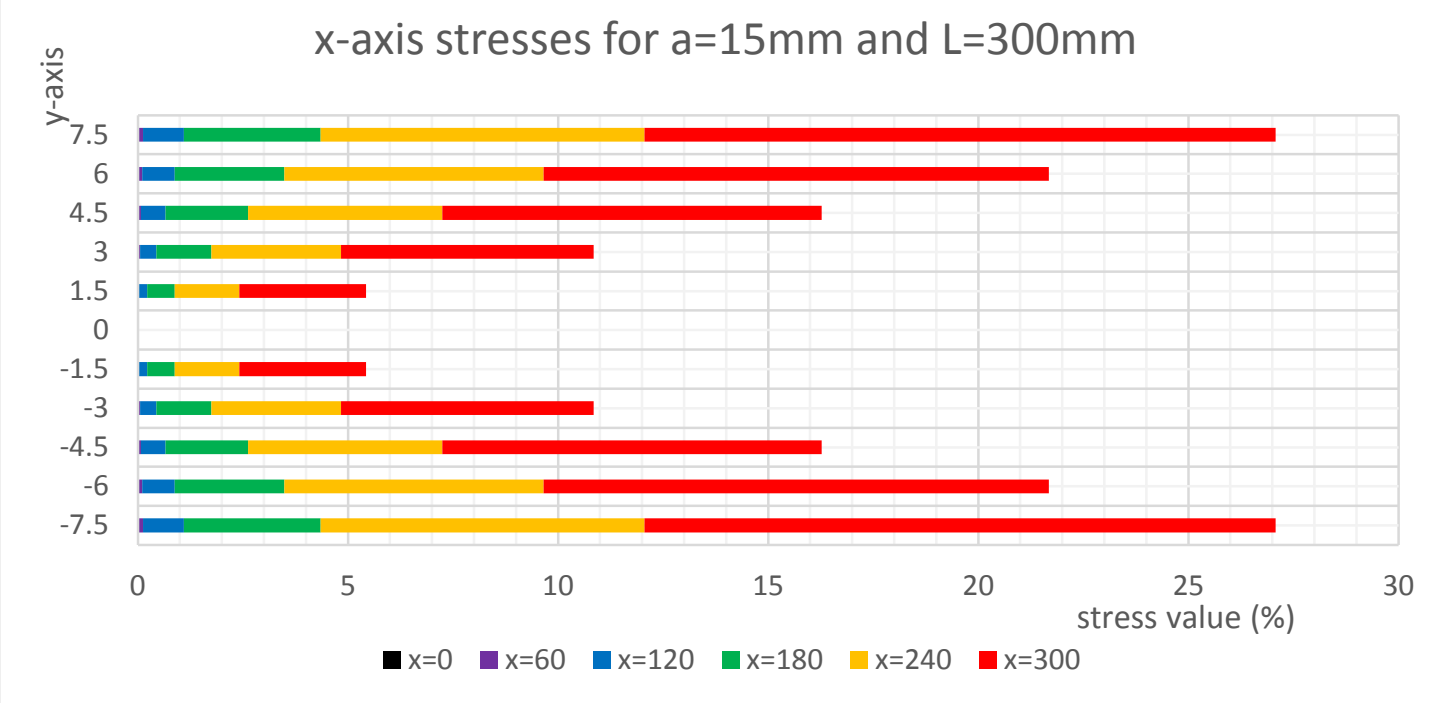

Fig. (5) $X$-Axis Stresses for $a=15 \mathrm{~mm}$ and $L=300 \mathrm{~mm}$

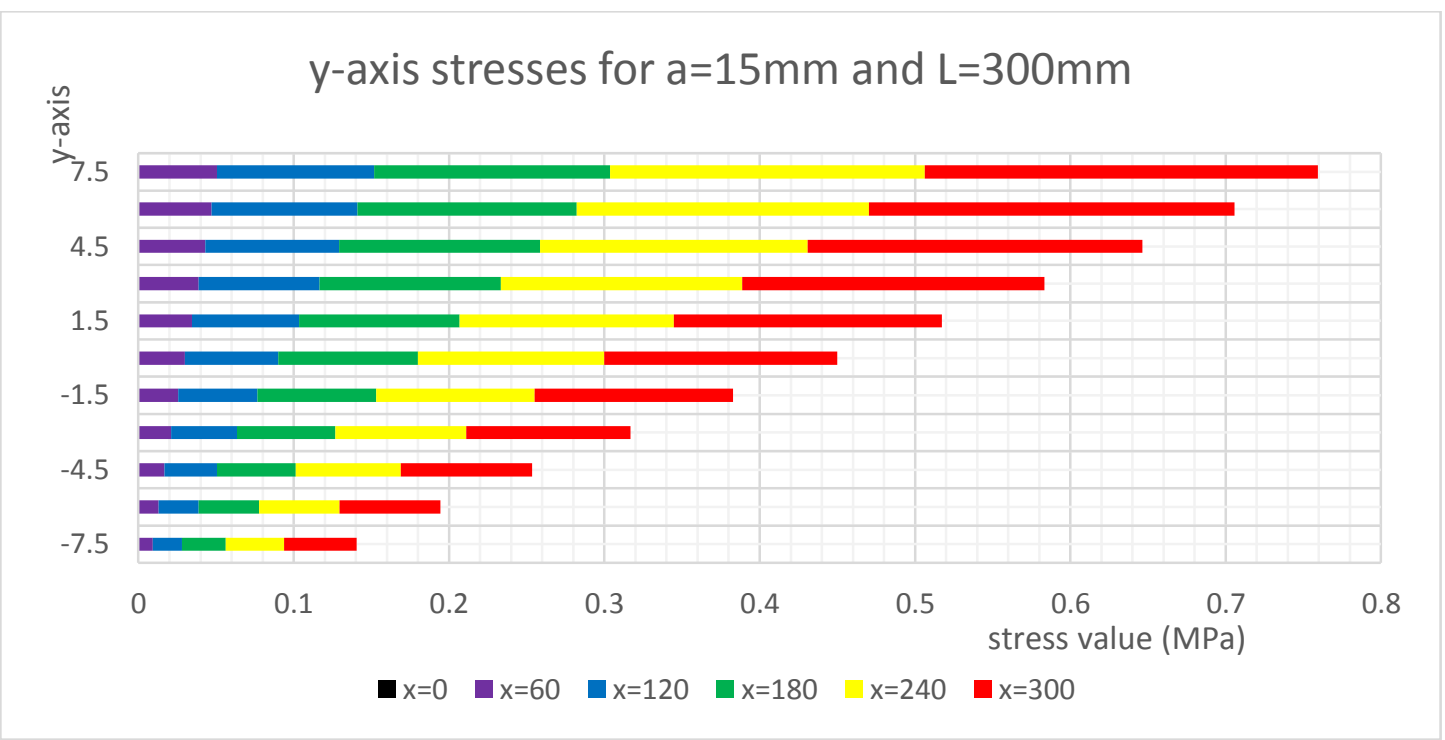

Fig. (6) Y-Axis Stresses for $\mathrm{a}=15 \mathrm{~mm}$ and $\mathrm{L}=300 \mathrm{~mm}$

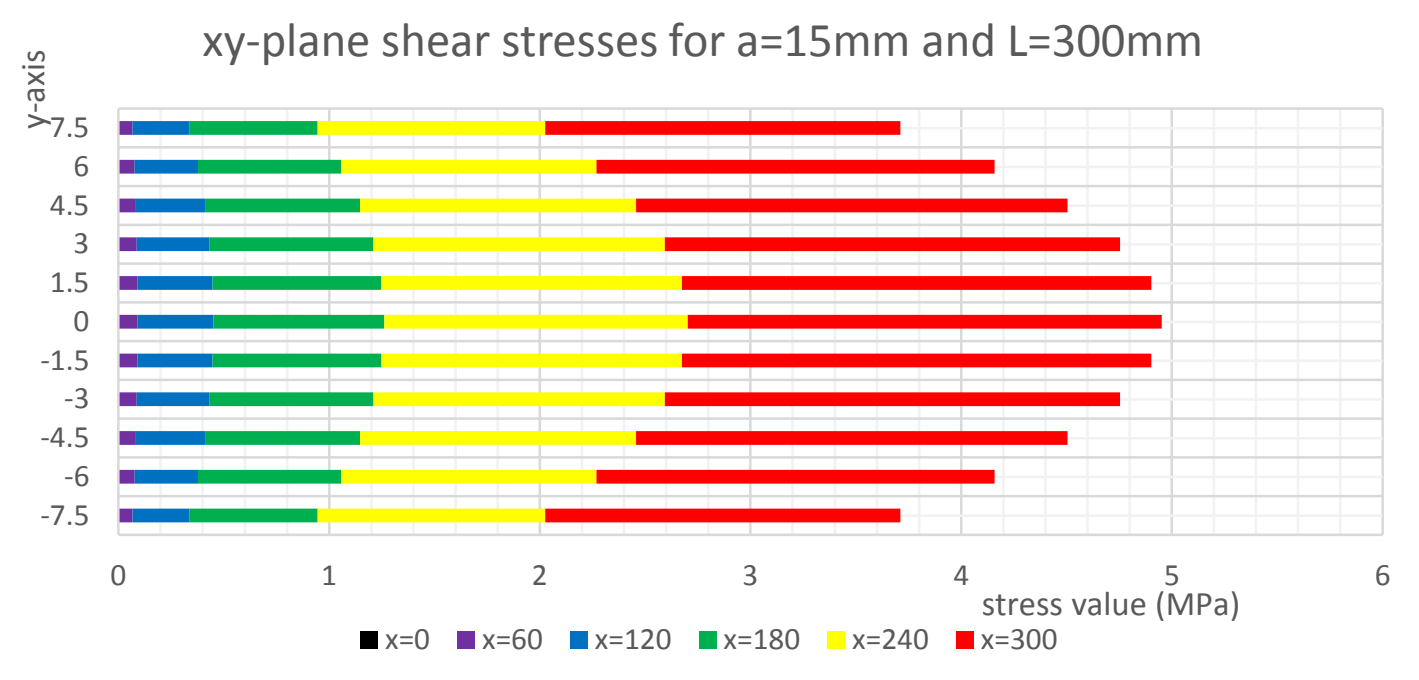

Fig. (7) XY-Plane Shear Stresses for $\mathrm{a}=15 \mathrm{~mm}$ and $\mathrm{L}=300 \mathrm{~mm}$ 


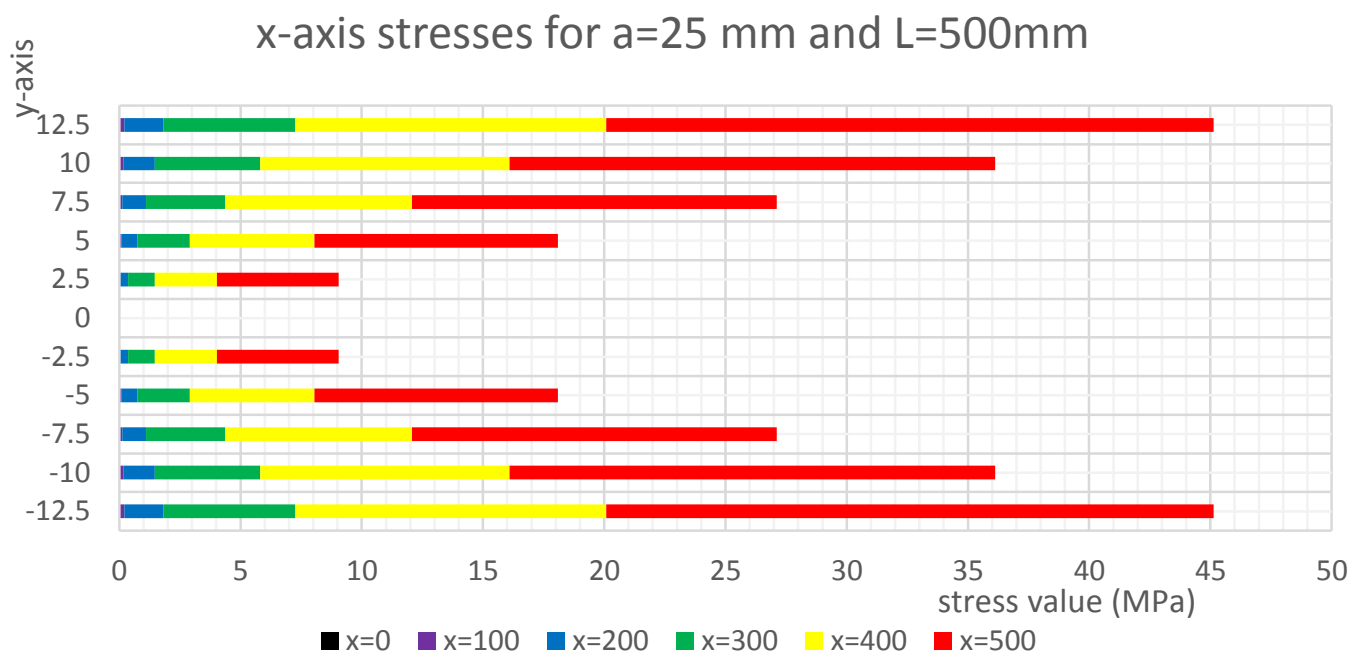

Fig. (8) $\mathrm{X}$-Axis Stresses for $\mathrm{a}=25 \mathrm{~mm}$ and $\mathrm{L}=500 \mathrm{~mm}$

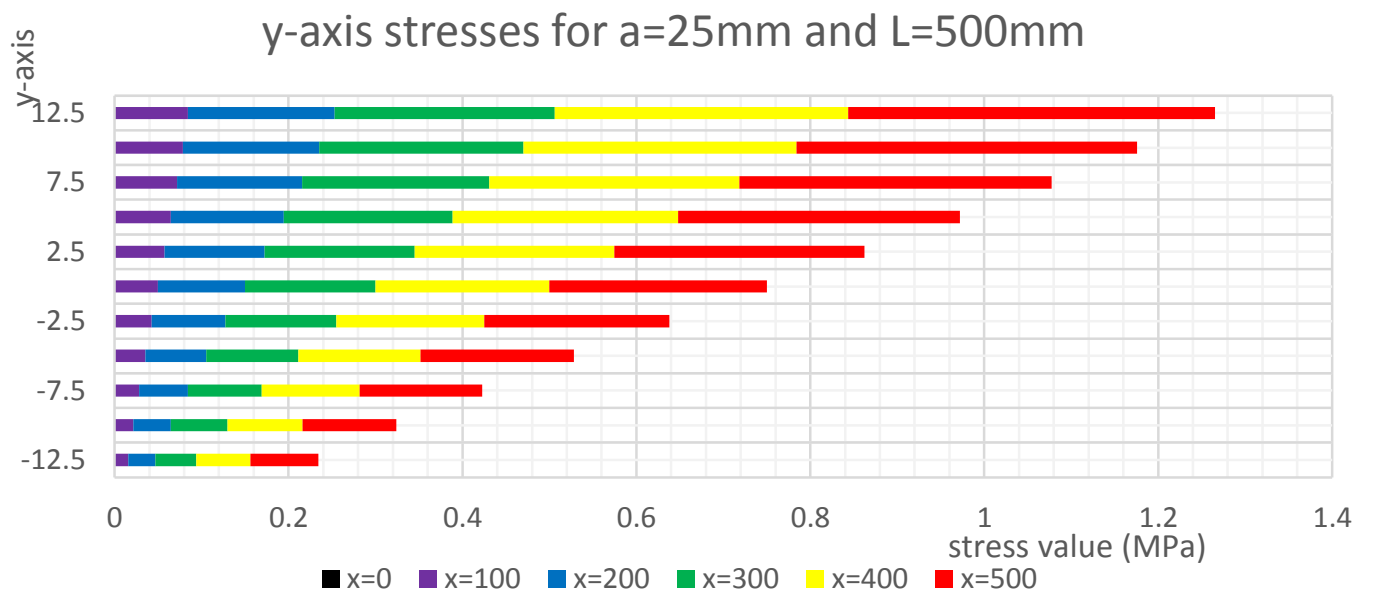

Fig. (9) Y-Axis Stresses for $\mathbf{a}=\mathbf{2 5 \mathrm { mm }}$ and $\mathrm{L}=500 \mathrm{~mm}$

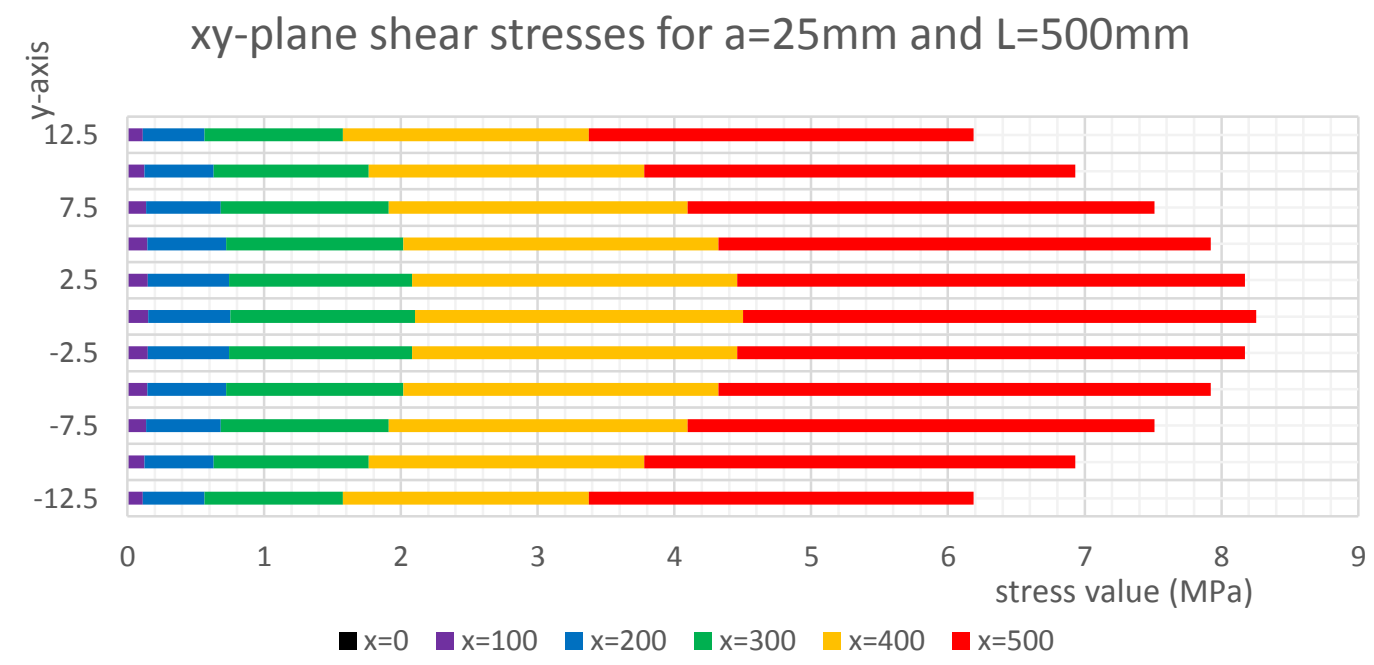

Fig. (10) XY-Plane Shear Stresses for $a=25 \mathrm{~mm}$ and $\mathrm{L}=500 \mathrm{~mm}$ 


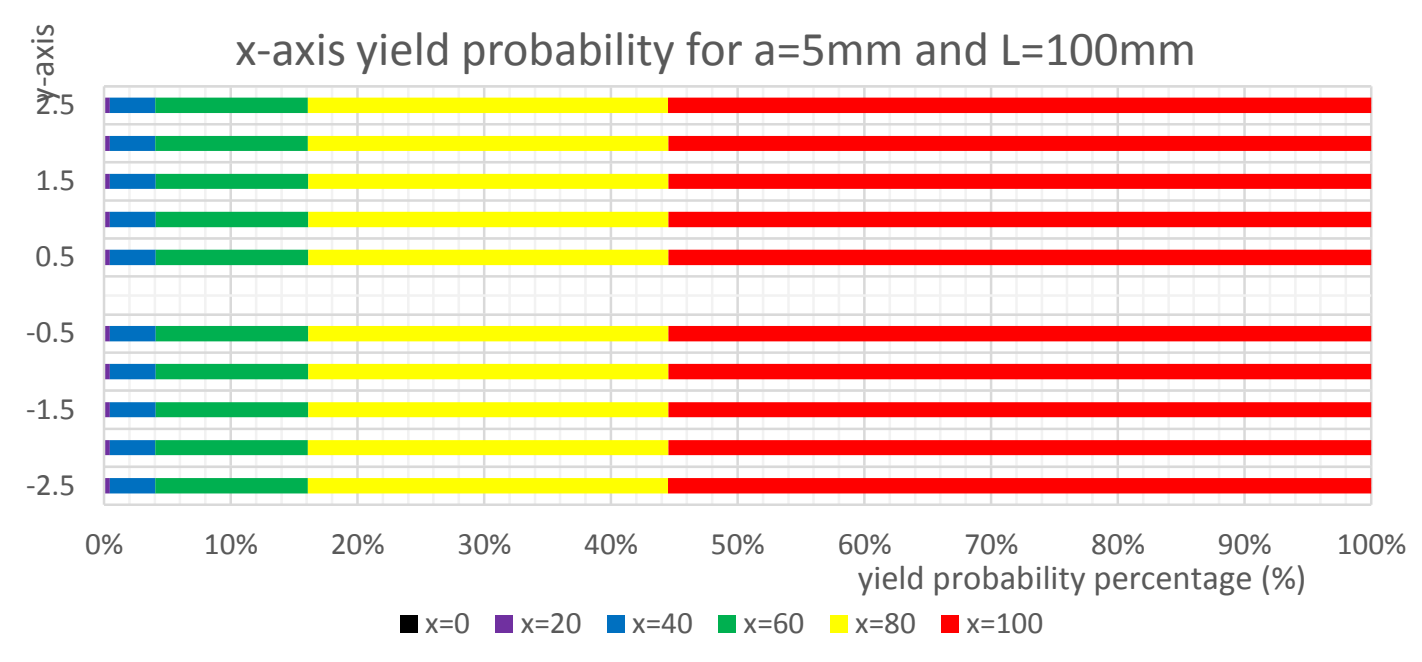

Fig. (11) X-Axis Yield Probability for $a=5 \mathrm{~mm}$ and $\mathrm{L}=100 \mathrm{~mm}$

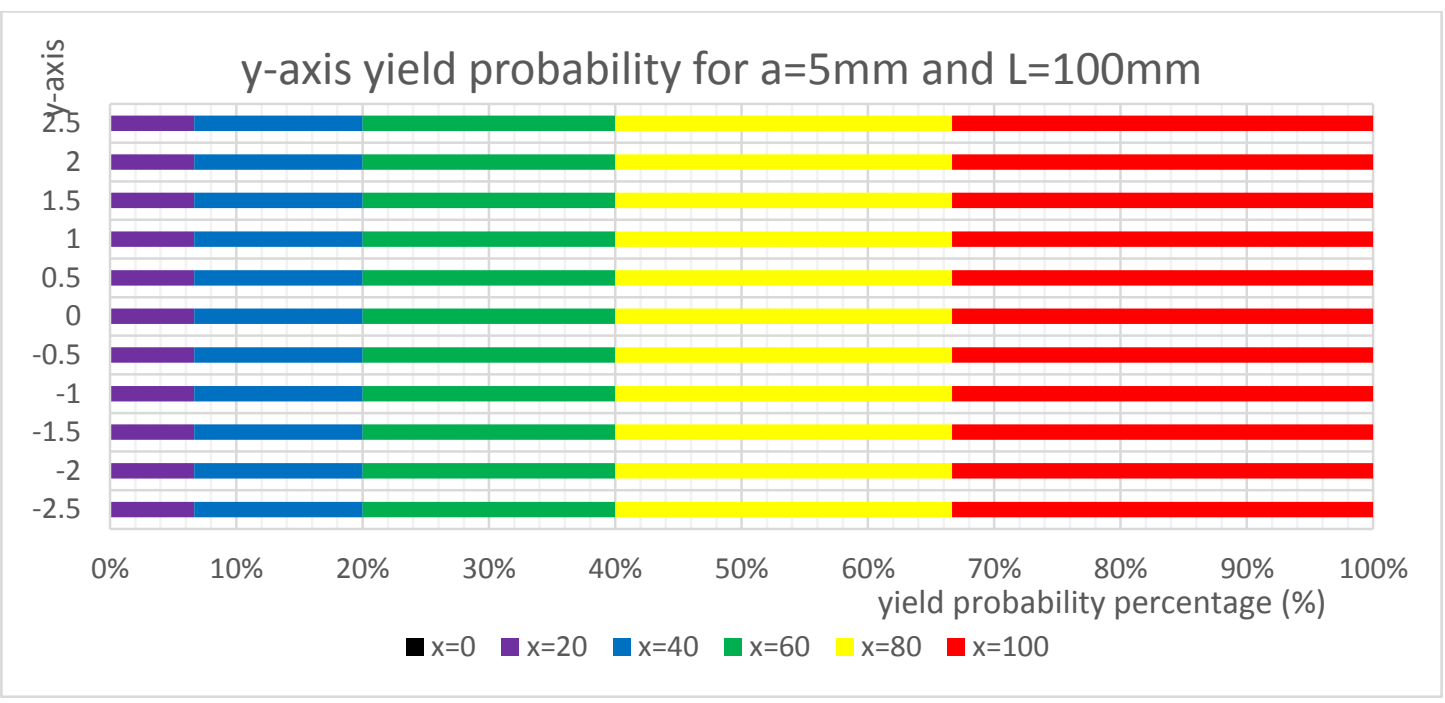

Fig. (12) Y-axis Yield Probability for $a=5 \mathrm{~mm}$ and $\mathrm{L}=100 \mathrm{~mm}$

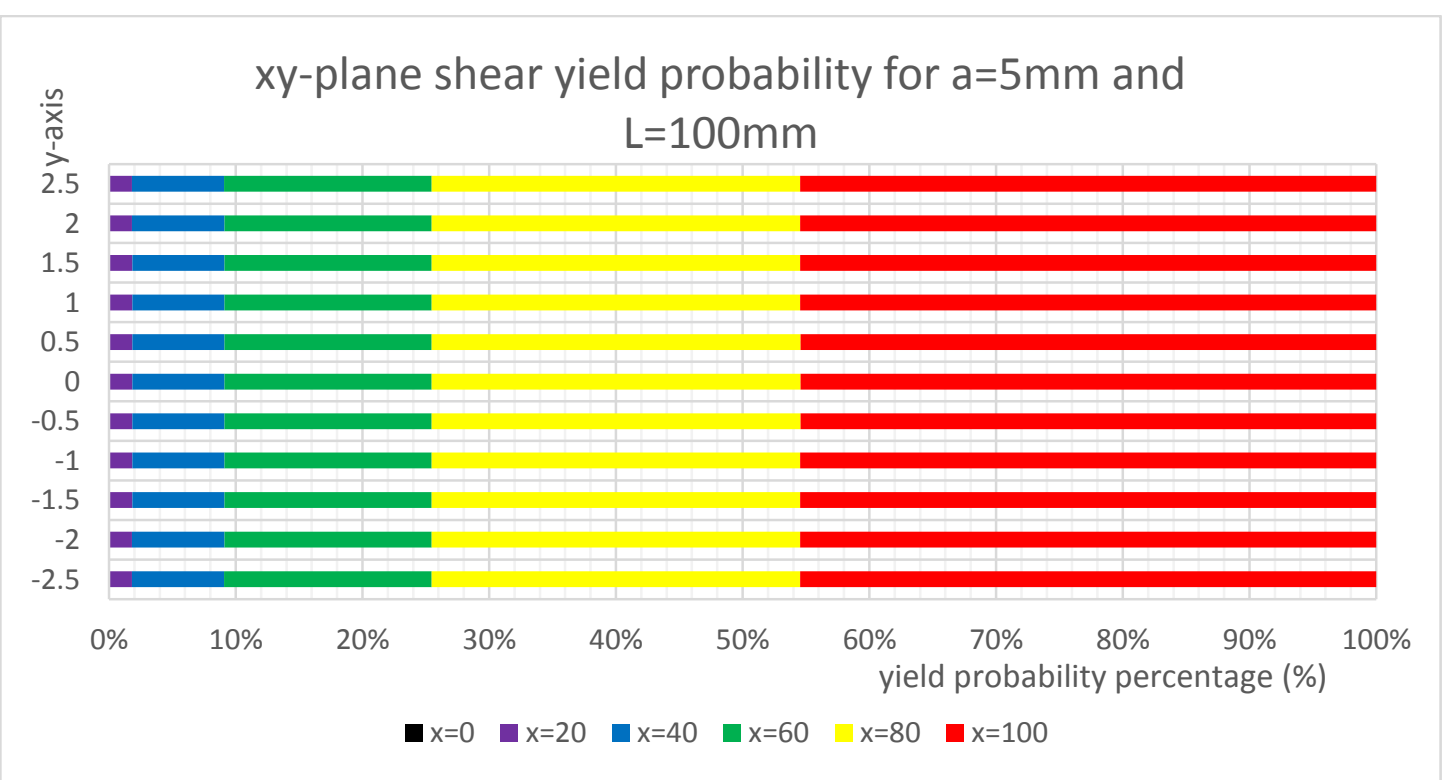

Fig. (13) XY-Plane Yield Probability for $\mathrm{a}=5 \mathrm{~mm}$ and $\mathrm{L}=100 \mathrm{~mm}$ 


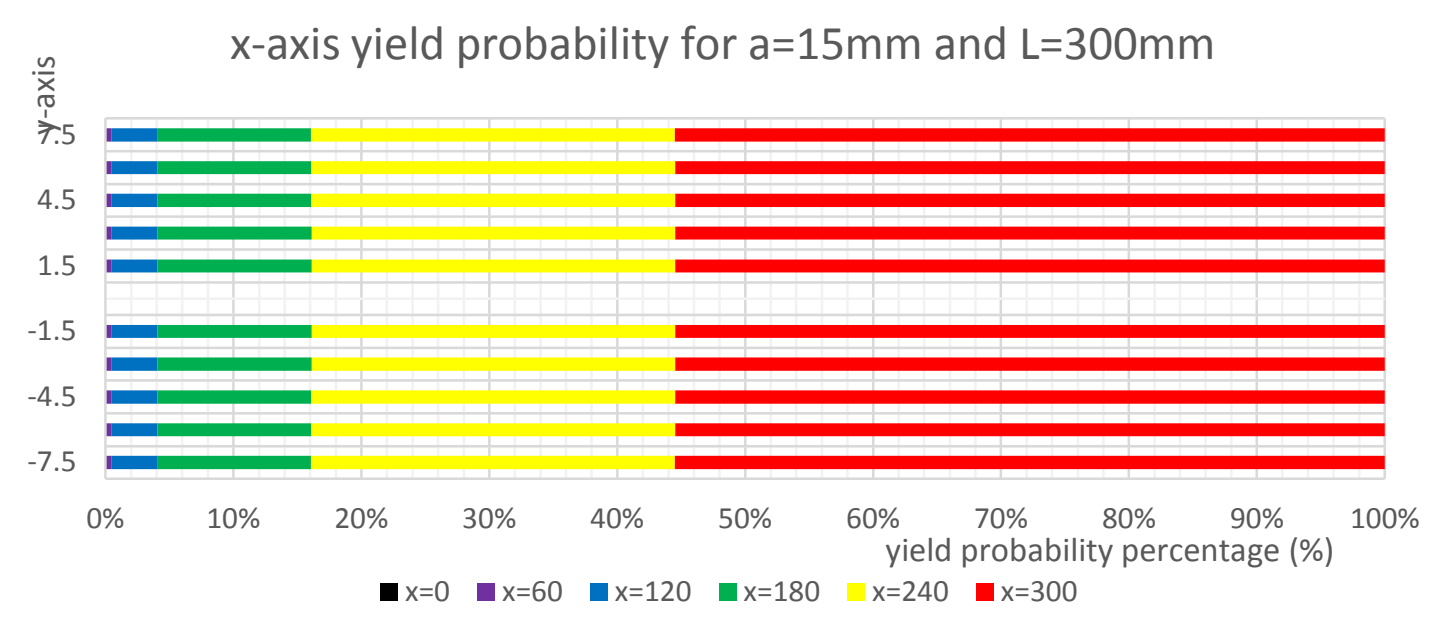

Fig. (14) $X$-Axis Yield Probability for $a=15 \mathrm{~mm}$ and $L=300 \mathrm{~mm}$

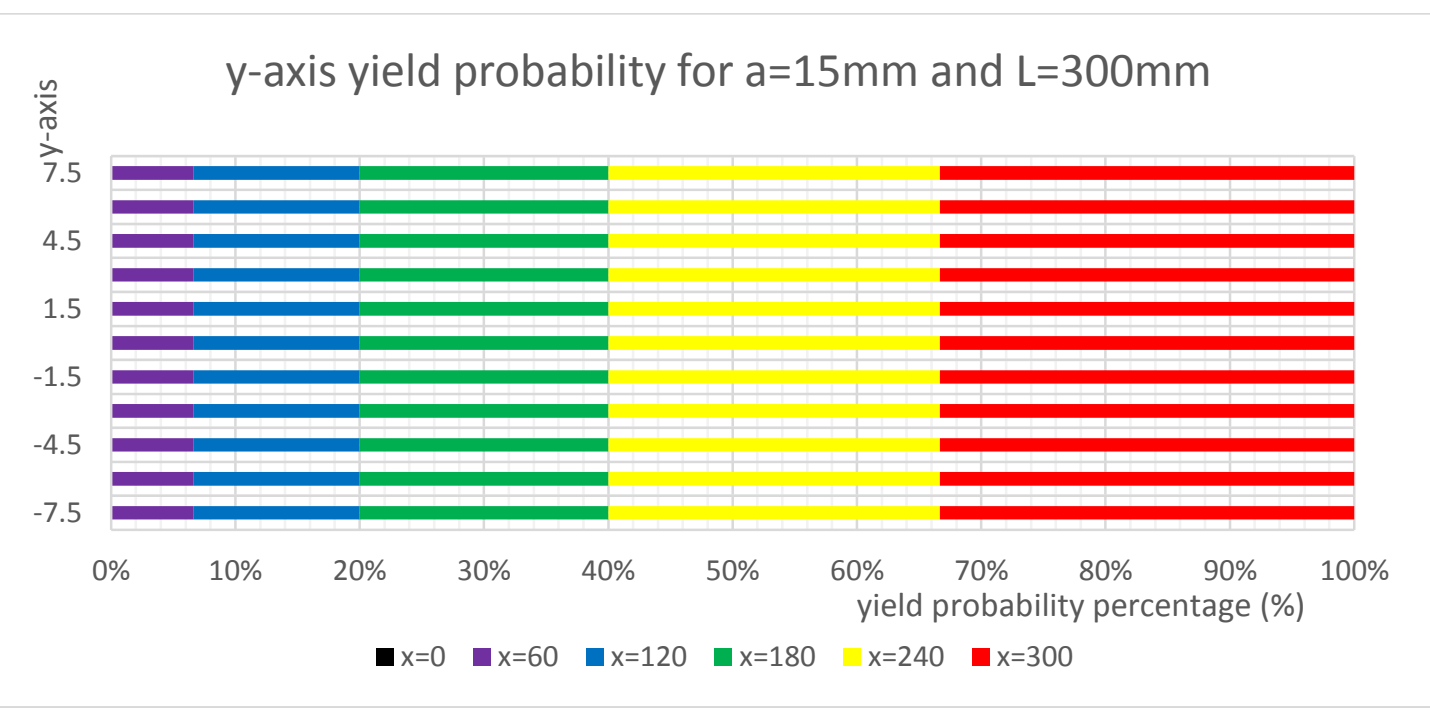

Fig. (15) Y-Axis Yield Probability for $a=15 \mathrm{~mm}$ and $L=300 \mathrm{~mm}$

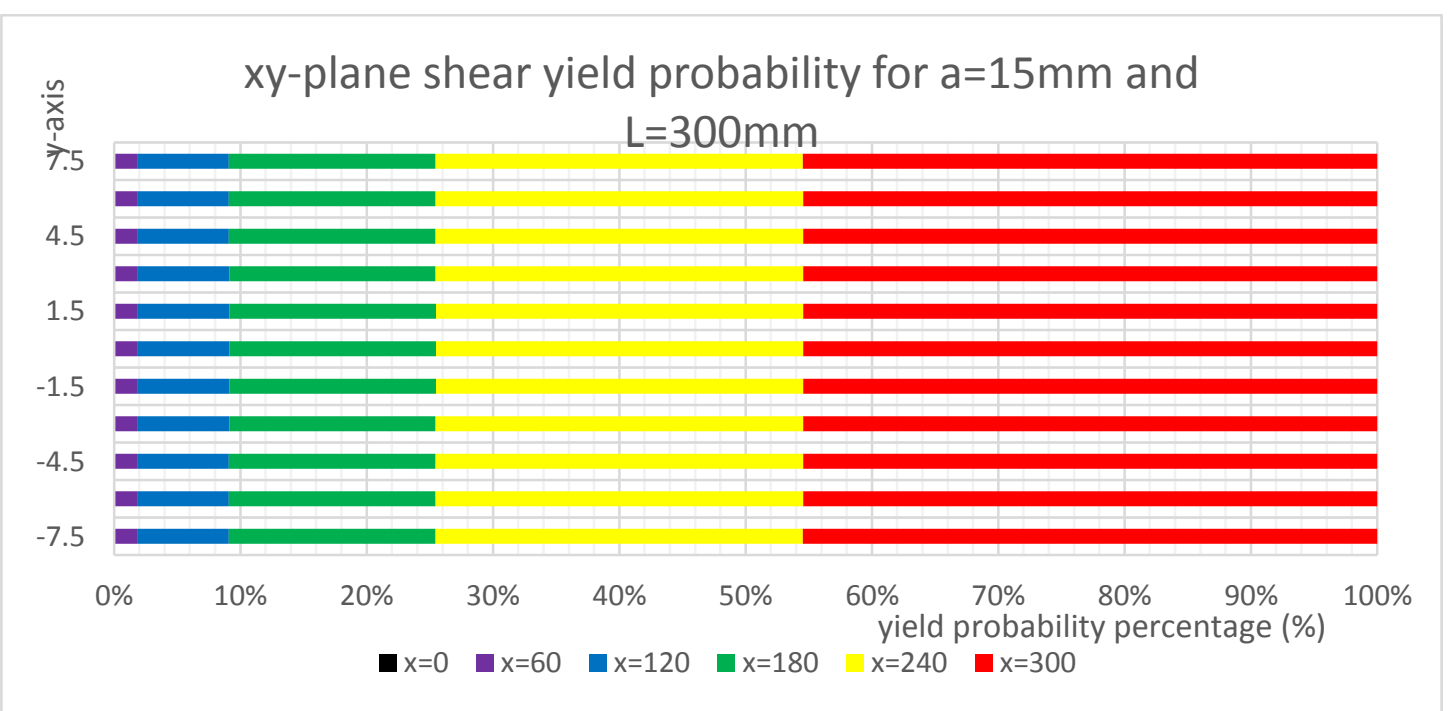

Fig. (16) XY-Plane Yield Probability for a=15mm and $L=300 \mathrm{~mm}$ 


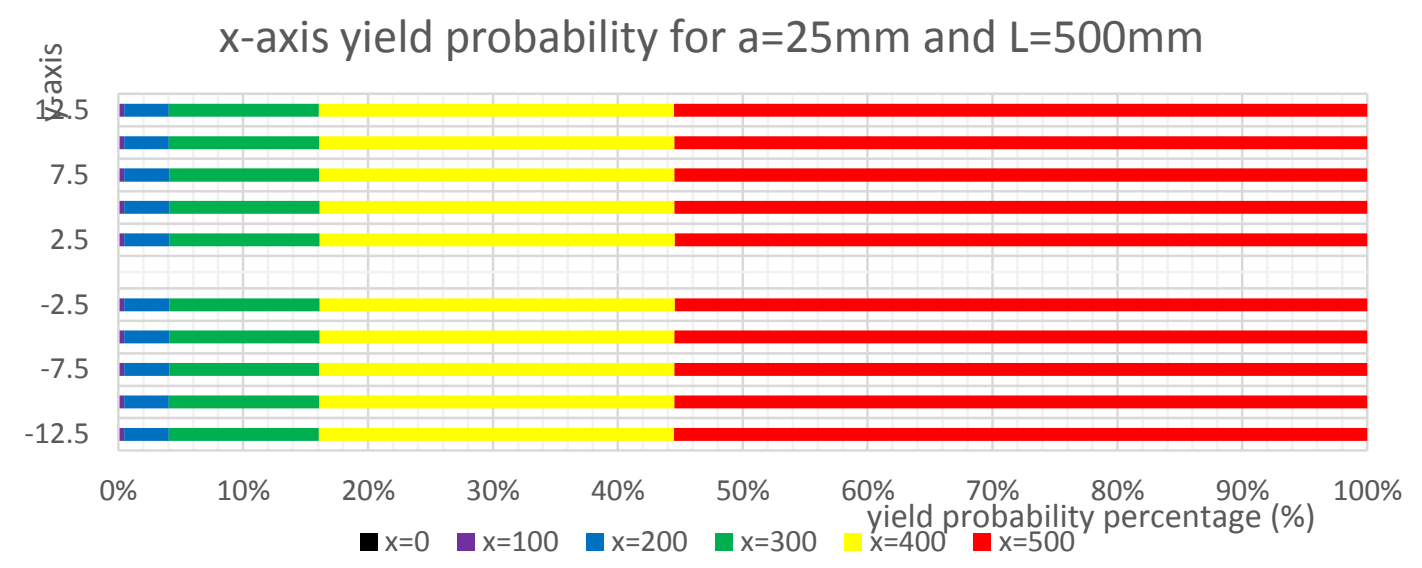

Fig. (17) X-Axis Yield Probability for a=25mm and $\mathrm{L}=500 \mathrm{~mm}$

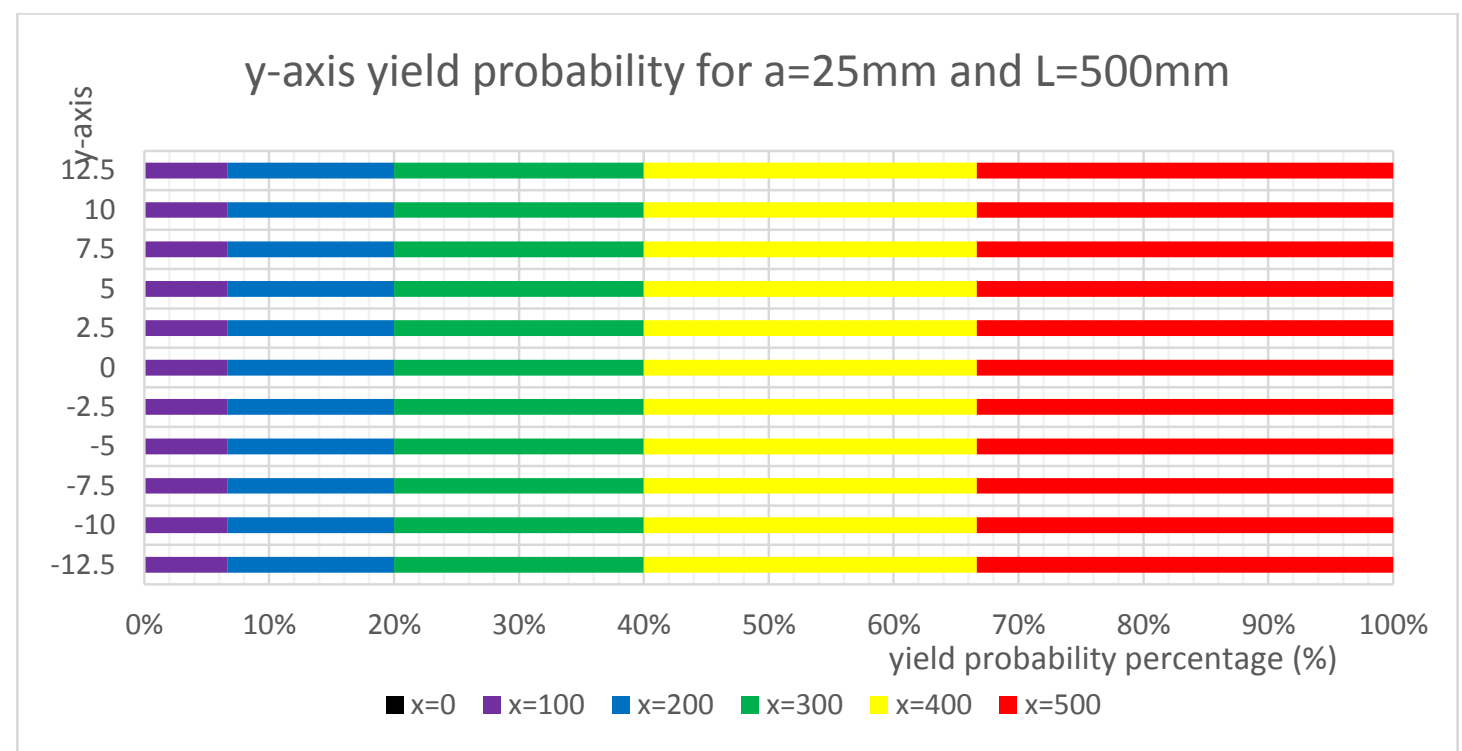

Fig. (18) Y-Axis Yield Probability for $a=25 \mathrm{~mm}$ and $\mathrm{L}=500 \mathrm{~mm}$

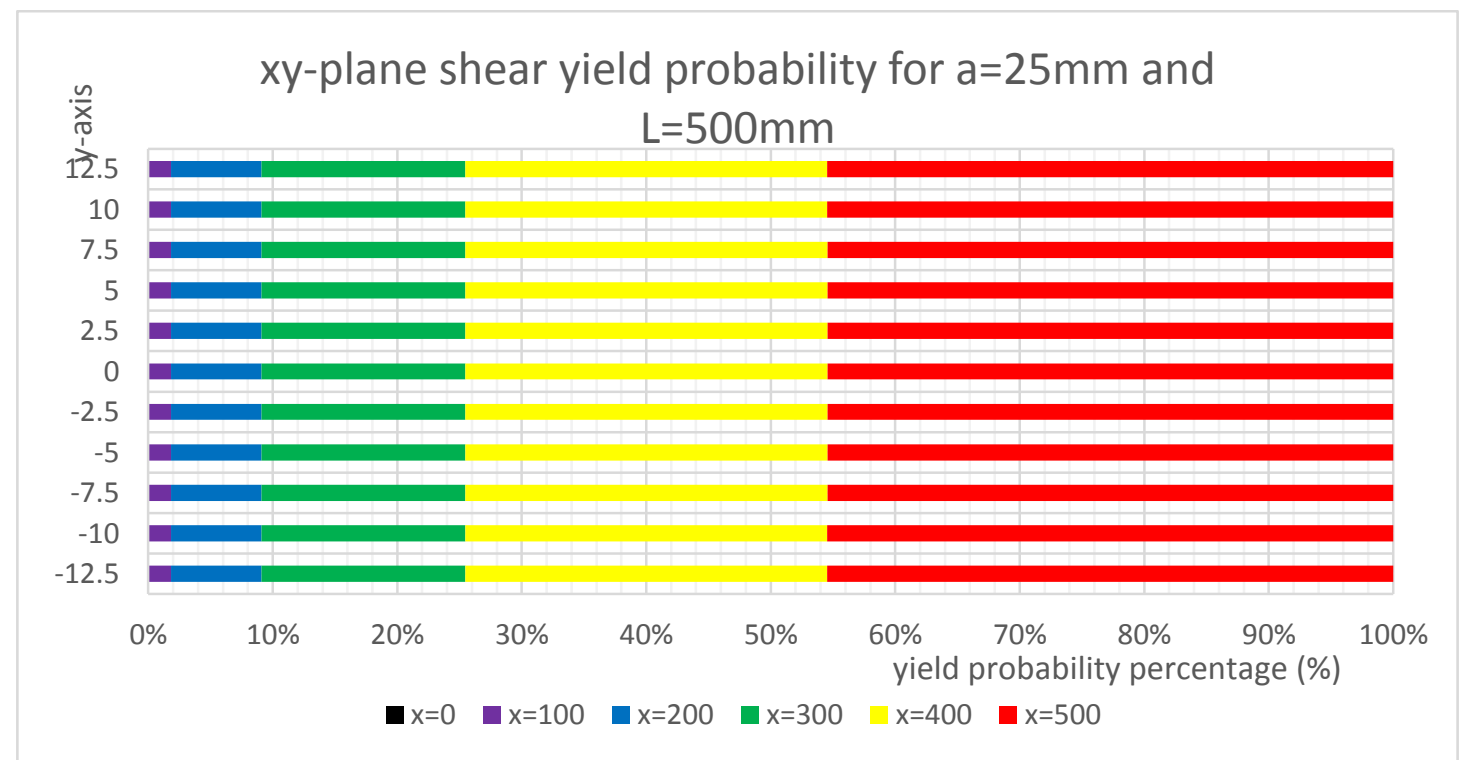

Fig. (19) XY-Plane Yield Probability for $a=25 \mathrm{~mm}$ and $\mathrm{L}=500 \mathrm{~mm}$ 


\section{References:}

[1] Yevick, D; 2003. Multicanonical Evaluation of Joint Probability Density Functions in Communications System Modeling; Photonics Technology Letters, IEEE, 15(11): 1540-1542

[2] Kim, C. H.; 2010. Airy Stress Function for Describing the NonLinear Effect of Simply-Supported Plates with Movable Edges; International Journal of Non-Linear Mechanics, 45(4): 395-408

[3] Hearn, E. J.; 1997. Mechanics of Materials 2: An introduction to the Mechanics of Elastic and Plastic Deformation of Solids and Structural
Materials. Third Edition; Butterworth Heinemann

[4] Waddy, D. S.; Chen, L.; Bao, X. 2004. Multicanonical Investigation of Joint Probability Density Function of PMD and PDL; Proceedings of SPIEISOE, 2(9): 831-839

[5] Larson, U. E.; Golaz, J. C.; Cotton, W. R. 2002. Small-scale and MesoScale Variability in Cloudy Boundary Layers: Joint Probability Density Functions; IEEE Science Power and Energy, 5(15): 732-751

[6] Spiegel, M. I., Schiller J. and Srinivasan R. A. 2009. Schaum Outlines of Probability and Statistics. Third Edition; Mc-Graw Hill Book Company

\section{طريقة توفيقية لحساب توزيع احتمالات الخضوع علي طول عتبة مثبتة من جانب واحل محملة بصورة خطية الخصوع علية
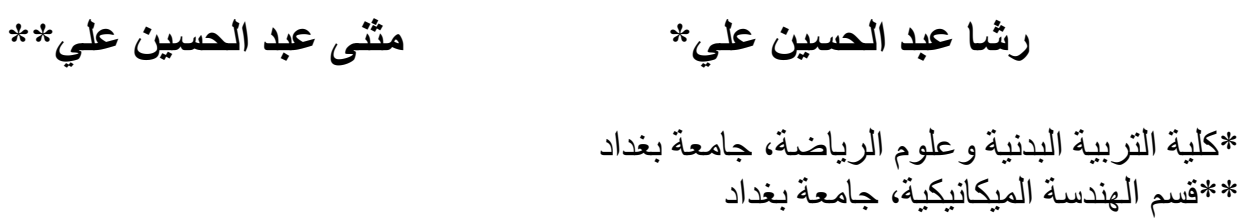

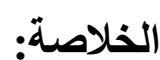

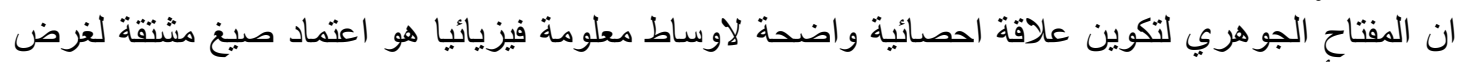

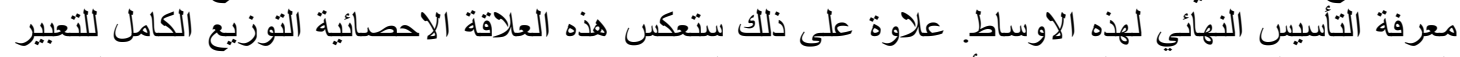

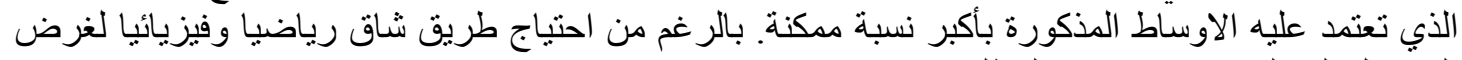

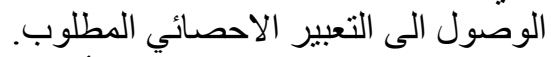

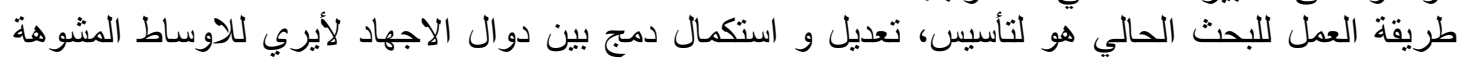

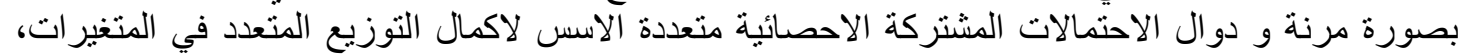

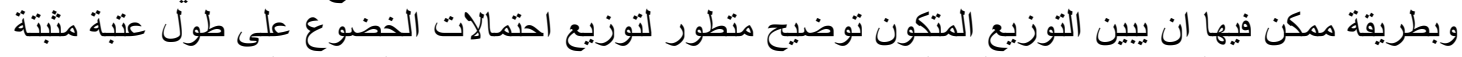

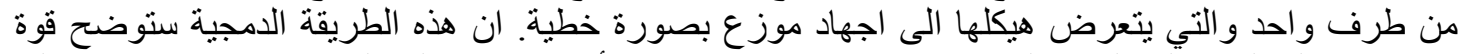

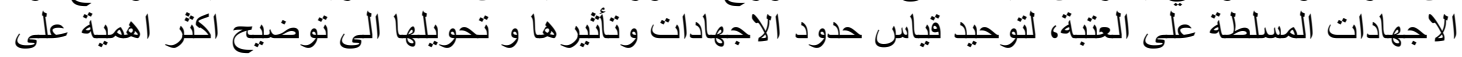

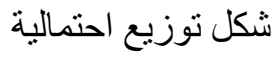

الكلمات المفتاحية: دوال الكثاقة الاحتمالية المشتركة متعددة المتغيرات، دوال الاحتمالات متعددة الاسس، دالة

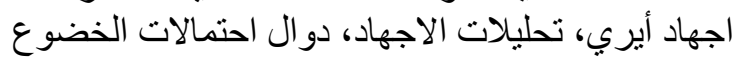

